\title{
Evaluation of Automatic Segmentation Model With Dosimetric Metrics for Radiotherapy of Esophageal Cancer
}

\author{
Ji Zhu ${ }^{\dagger}$, Xinyuan Chen ${ }^{\dagger}$, Bining Yang, Nan Bi, Tao Zhang, Kuo Men* and Jianrong Dai* \\ National Cancer Center/National Clinical Research Center for Cancer/Cancer Hospital, Chinese Academy of Medical \\ Sciences and Peking Union Medical College, Beijing, China
}

\section{OPEN ACCESS}

Edited by:

An Liu,

City of Hope National Medical Center,

United States

Reviewed by:

Chengyu Shi,

St. Vincent's Medical Center,

United States

Dongsu Du,

City of Hope National Medical Center

United States

Richard Li,

City of Hope National Medical Center

United States

${ }^{*}$ Correspondence:

Jianrong Dai

dai_jianrong@cicams.ac.cn

Kuo Men

menkuo@cicams.ac.cn

tThese authors have contributed equally to this work and share co-first authorship

Specialty section:

This article was submitted to

Radiation Oncology,

a section of the journal

Frontiers in Oncology

Received: 22 May 2020

Accepted: 17 August 2020

Published: 29 September 2020

Citation:

Zhu J, Chen X, Yang B, Bi N, Zhang T, Men K and Dai J (2020) Evaluation of Automatic Segmentation Model With Dosimetric Metrics for Radiotherapy of Esophageal Cancer.

Front. Oncol. 10:564737.

doi: 10.3389/fonc.2020.564737
Background and Purpose: Automatic segmentation model is proven to be efficient in delineation of organs at risk (OARs) in radiotherapy; its performance is usually evaluated with geometric differences between automatic and manual delineations. However, dosimetric differences attract more interests than geometric differences in the clinic. Therefore, this study aimed to evaluate the performance of automatic segmentation with dosimetric metrics for volumetric modulated arc therapy of esophageal cancer patients.

Methods: Nineteen esophageal cancer cases were included in this study. Clinicians manually delineated the target volumes and the OARs for each case. Another set of OARs was automatically generated using convolutional neural network models. The radiotherapy plans were optimized with the manually delineated targets and the automatically delineated OARs separately. Segmentation accuracy was evaluated by Dice similarity coefficient (DSC) and mean distance to agreement (MDA). Dosimetric metrics of manually and automatically delineated OARs were obtained and compared. The clinically acceptable dose difference and volume difference of OARs between manual and automatic delineations are supposed to be within 1 Gy and $1 \%$, respectively.

Results: Average DSC values were greater than 0.92 except for the spinal cord (0.82), and average MDA values were $<0.90 \mathrm{~mm}$ except for the heart $(1.74 \mathrm{~mm})$. Eleven of the 20 dosimetric metrics of the OARs were not significant $(P>0.05)$. Although there were significant differences $(P<0.05)$ for the spinal cord (D2\%), left lung $(\mathrm{V} 10, \mathrm{~V} 20, \mathrm{~V} 30$, and mean dose), and bilateral lung (V10, V20, V30, and mean dose), their absolute differences were small and acceptable for the clinic. The maximum dosimetric metrics differences of OARs between manual and automatic delineations were $\Delta \mathrm{D} 2 \%=0.35 \mathrm{~Gy}$ for the spinal cord and $\Delta \mathrm{V} 30=0.4 \%$ for the bilateral lung, which were within the clinical criteria in this study.

Conclusion: Dosimetric metrics were proposed to evaluate the automatic delineation in radiotherapy planning of esophageal cancer. Consequently, the automatic delineation could substitute the manual delineation for esophageal cancer radiotherapy planning based on the dosimetric evaluation in this study.

Keywords: automatic segmentation, dosimetric evaluation, esophageal cancer, deep learning, organs at risk, radiotherapy 


\section{INTRODUCTION}

One of the challenges in radiotherapy is the accurate delineation of organs at risk (OARs). Various delineation techniques are used by different professionals. Automatic segmentation of OARs with artificial intelligence has great application value for treatment planning in radiotherapy because of its high efficiency and advanced delineation accuracy.

Several studies focused on the geometric evaluation between manual and automatic segmentation delineations. The geometric evaluation compares the similarity between different delineation methods by Dice similarity coefficient (DSC) and mean distance to agreement (MDA). The DSC evaluates the similarity of two delineations by comparing the overlap area. The MDA shows the average distance of outline points between the overlap volume of two delineations. Liang et al. (1) evaluated the quality of automatic delineation by using geometric discrepancies in head and neck OARs. Ahn et al. (2) demonstrated that the deep convolution neural network methods could provide an effective and efficient way to delineate OARs for liver cancer. For automatic delineation in the thorax, Yang et al. (3) reported that several institutions participated in the thoracic automatic segmentation challenge organized by the American Association of Physicists in Medicine in 2017. The DSC scores of the left lung, right lung, heart, and spinal cord were $0.956 \pm 0.019,0.955 \pm 0.019,0.931 \pm 0.015$, and $0.862 \pm 0.038$, respectively (3). Lustberg et al. (4) showed their geometric evaluation of automatic delineations for lung cancer in 2018: the spinal cord (median Dice score, 0.83), the lungs (median Dice score, >0.95), and the heart (median Dice score, >0.90). Dong et al. (5) addressed that the averaged DSC scores for the left lung, right lung, spinal cord, and heart were 0.97, $0.97,0.90$, and 0.87 , correspondingly, in 2019. Therefore, thoracic OARs including the spinal cord, lungs, and heart could be segmented accurately by the automatic delineation method (5).

However, the primary concern in radiotherapy is not the delineation accuracy but the dosimetric impacts of the delineation. To show that a model successfully segments the OARs in geometry is not sufficient to confirm its reliability for radiotherapy utilization. Vinod et al. (6) believed that it is similar to geometric evaluation of different delineations; there was no standardized method of dosimetric comparison of delineations. Accordingly, a quantitative system to evaluate both the dosimetric and geometric parameters of manual and automatic delineation-generated plans becomes necessary. Fung et al. (7) showed their studies about geometric discrepancies and dose impact between manually and automatically delineated OARs in nasopharyngeal carcinoma in a creative manner. Especially, Fung et al. (7) evaluated manual and automatic delineations using dosimetric discrepancies, which include

Abbreviations: OARs, Organs at risk; VMAT, Volumetric modulated arc therapy; CNN, Convolutional neural network; DSC, Dice similarity coefficient; MDA, Mean distance to agreement; DCNN, Deep convolution neural network; CT, Computed tomography; PTV, Planning target volume; PGTV, Planning gross tumor volume; PRV, Planning organ at risk volume; DVH, Dose volume histogram.
TABLE 1 | The Clinical data of patients.

\begin{tabular}{|c|c|c|}
\hline Characteristic & & $N(n=19)$ \\
\hline Age & Median & $59.05 \pm 8.26$ \\
\hline \multirow[t]{2}{*}{ Gender } & Male & 16 \\
\hline & Female & 3 \\
\hline \multirow[t]{2}{*}{ Pathology } & Squamous cell carcinoma & 18 \\
\hline & Small cell carcinomas & 1 \\
\hline \multirow[t]{2}{*}{ Primary location } & Cervical & 0 \\
\hline & Thoracic & 19 \\
\hline \multirow[t]{3}{*}{ Chemotherapy } & Neoadjuvant & 8 \\
\hline & Concurrent & 8 \\
\hline & None & 3 \\
\hline \multirow[t]{2}{*}{ T stage } & T3 & 11 \\
\hline & T4 & 8 \\
\hline \multirow[t]{3}{*}{ N stage } & N1 & 17 \\
\hline & N2 & 1 \\
\hline & N3 & 1 \\
\hline \multirow[t]{2}{*}{ M stage } & MO & 14 \\
\hline & M1 & 5 \\
\hline
\end{tabular}

The values in the "Age" row represent as mean \pm standard deviation.

maximum dose, D1 cc, and D50\%. However, their study did not evaluate the automatic delineation using clinical dosimetric metrics.

No study on the impact of dosimetric metrics between manual and automatic delineations has been conducted yet, specifically in esophageal cancer. Further, esophageal cancer is common around Asia, especially in eastern Asia. More than 700 esophageal cancer patients are treated by radiotherapy in our department every year. Therefore, automatic delineation of esophageal cancer will play an important role in the clinic. This study introduces a dosimetric evaluation method to substitute the geometric evaluations on automatic delineation for esophageal cancer VMAT radiotherapy.

\section{METHODS}

\section{Data Acquisition}

The data consisted of 19 stage III/IV esophageal cancer patients who were treated from December 2018 to July 2019 in our department. The inclusion criteria of patients were proven and diagnosed histologically as esophageal cancer according to the guideline of the TNM staging system. The detailed demographics of the included patients are shown in Table 1. All patients were set up with the supine position on a commercial "bellyboard" and immobilized using a thermoplastic mask. The data of planning computed tomography (CT) images were acquired from the Somatom Definition AS 40 (Siemens Healthcare, Forchheim, Germany) or the Brilliance CT Big Bore (Philips Healthcare, Best, the Netherlands) systems on helical scan mode. CT images were reconstructed using a matrix size of $512 \times 512$ and a slice thickness of $5 \mathrm{~mm}$. The delineation of OARs was delineated 


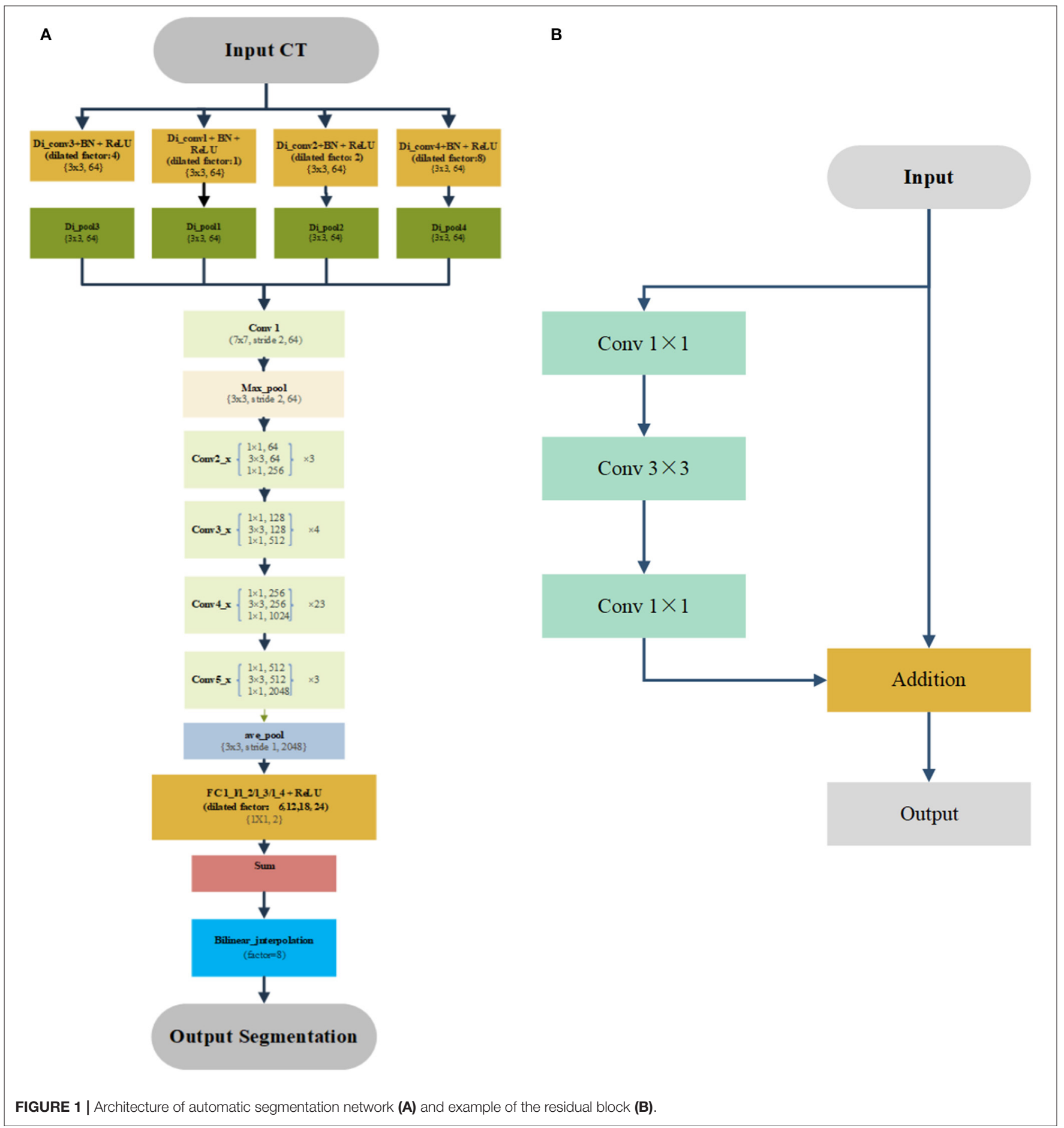

on CT images according to RTOG 0617 and RTOG 1106 standard contouring atlas $(8,9)$. Meanwhile, the delineation of OARs was delineated and approved by senior clinicians for this study.

\section{Architecture of Segmentation Network}

Five hundred patients diagnosed with thorax tumor who received radiotherapy between 2011 and 2019 were enrolled for training the OAR-segmentation models. The OARs for segmentation included bilateral lungs, heart, spinal cord, and bilateral kidneys. Fifty patients from 2018 to 2019 were chosen randomly to validate the deep learning model. The validation set was used to assess the performance of the deep learning model.

We used this previously published deep learning algorithm to segment the OARs for treatment planning (10). Figure 1 shows 
the detailed architectures. A four-stream dilated convolutional module was applied before the ResNet-101 networks. The advantage is that it can extract multiscale features from the original CT image with different dilated factors. The multiscale feature maps were added and feed forward to the ResNet101, which has 101 weighted layers. Its characteristic is the use of several residual blocks to avoid gradients vanishing. An example of the residual block is shown in Figure 1B. It took a standard feed-forward convolutional network and added skipped connections that bypassed a few convolutional layers at a time. Each bypass gave rise to a residual block in which the convolutional layers predicted a residual that was added to the input tensor of the block. There were $3,4,23$, and 4 such blocks in conv2, conv3, conv4, and conv5, respectively. The size of image was reduced to $1 / 8$ of the original network with the down-sampling. Therefore, a bilinear interpolation was applied to the sum layer to recover the feature map to the original size for pixellevel classification.

\section{Experiments}

The clinicians manually delineated the planning target volume, the planning gross tumor volume, and the OARs, including the spinal cord, spinal cord planning OAR volume (PRV), heart, left lung, right lung, and whole lung, as the ground truth (GT) set. The previously published deep learning model was used for this automatic segmentation task (10). The automatically delineated OARs included the spinal cord, spinal cord PRV, heart, left lung, right lung, and bilateral lung.

The work flowchart of this study is illustrated in Figure 2. The radiotherapy plans were designed and optimized with the manually delineated targets and the automatically delineated OARs. The dose constraints are followed by published guideline: the maximum dose of the spinal cord was $\leq 40 \mathrm{~Gy}$, the spinal cord PRV was $\leq 45$ Gy, V20 Gy of the bilateral lung was $\leq 25$ or $30 \%$ in special cases, and the heart V30 and V40 Gy was $\leq 40$ and $\leq 30 \%$, respectively $(8,11)$. D $(x \%)$ means of the dose received by $x \%$ of the OARs volume. Dmean is defined as the average dose of OARs receiving. The $\mathrm{V} x \mathrm{~Gy}$ is defined as the volume of normal OARs receiving more than $x$ Gy dose (10). Further, the clinically acceptable dose difference and volume difference of OARs between manual and automatic delineations should be $<1$ Gy and $1 \%$, respectively. All the plans were evaluated and approved by senior clinicians.

Next, the dosimetric metrics of the plans were calculated and evaluated using the manual and automatic segmentation delineated OARs, separately. Finally, both manual and automatic delineations were compared with metrics of the geometry and clinical dosimetry.

\section{Evaluation}

\section{Geometric Metrics}

The DSC and MDA were used in this study $(7,12,13)$.

As shown above, the DSC is one of the geometric evaluation methods in this study, otherwise known as Sørensen-Dice coefficient (14), which is used to evaluate the similarity of two samples such as imaging and radiotherapy target volume segmentation.

$$
\operatorname{DSC}(\mathrm{A}, \mathrm{B})=\frac{2|\mathrm{~A} \cap \mathrm{B}|}{|\mathrm{A}|+|\mathrm{B}|}
$$

The DSC had values between 0 and $1(0=$ no overlap, $1=$ complete overlap). $A$ is the investigator (automatic) delineation, and $B$ is the GT (manual) delineation.

The MDA indicates the average distance of outline points of the automatic contouring volume to the outline of reference manual delineation perfect overlap volume (15). The lower the values $(\mathrm{mm})$ of MDA, the higher the correspondence between the automatic and manual contouring volumes (15).

\section{Dosimetric Metrics}

Radiotherapy plans were designed by using the Pinnacle $\left.{ }^{3(}\right)$ Radiation Therapy Planning software (version 9.1; Philips Medical Systems Inc., Fitchburg, MA, USA). The dosimetric parameters, including D2\%, Dmean, V40, V30, V20, and V10 Gy, were used to evaluate the plan quality and OARs sparing.

The continuous variables were presented as the mean \pm SD and should be rounded up to two decimal places, which are dependent on the normality of the data. Correspondingly, the paired $t$-test was used to compare the variables between the manually and automatically delineated methods. All of the statistical analyses were conducted using the IBM SPSS Statistics software (version 25.0; IBM Inc., Armonk, NY, USA). All paired $t$-tests were two-sided. The difference between manually (GT) and automatically delineated dosimetric metrics was considered as statistically significant when the paired $t$-test showed $P<0.05$.

The dosimetric characteristics of OARs were gauged by the conformity index (CI) and homogeneity index (HI) $(11,16,17)$. CI of target volume is defined as following equation (11):

$$
\mathrm{CI}=\frac{\mathrm{TVPTV}^{2}}{\mathrm{TV} * \mathrm{PTV}}
$$

where TV is the volume of prescribed isodose line enclosed volume. PTV is the volume of targets. TVPTV represents the overlap volume between volume of targets volume and the prescribed isodose line enclosed volume.

$\mathrm{HI}$ of target volume is a simple scoring tool that quantifies dose homogeneity in the target volume. It is therefore used to evaluate and compare the dose distributions of various treatment plans $(11,17)$.

The formula of HI is suggested by the ICRU 83 report as the following equation:

$$
\mathrm{HI}=\frac{\mathrm{D} 2 \%-\mathrm{D} 98 \%}{\mathrm{D} 50 \%}
$$

The D2\%, D98\%, and D50\% are doses delivered to 2, 98, and 50\% volume of target volume, respectively. The closer the HI value approaches 0 , the better homogeneity of target volume is (11). 


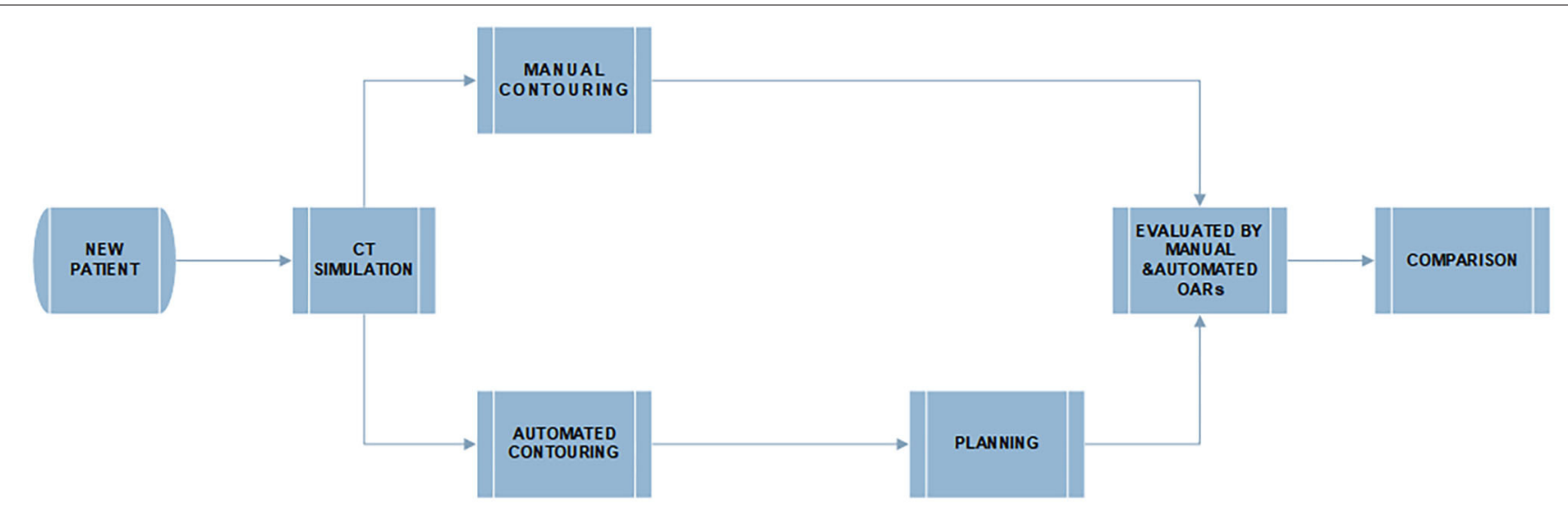

FIGURE 2 | The flowchart of manual and deep learning automatic delineation-based plan evaluation experiment.

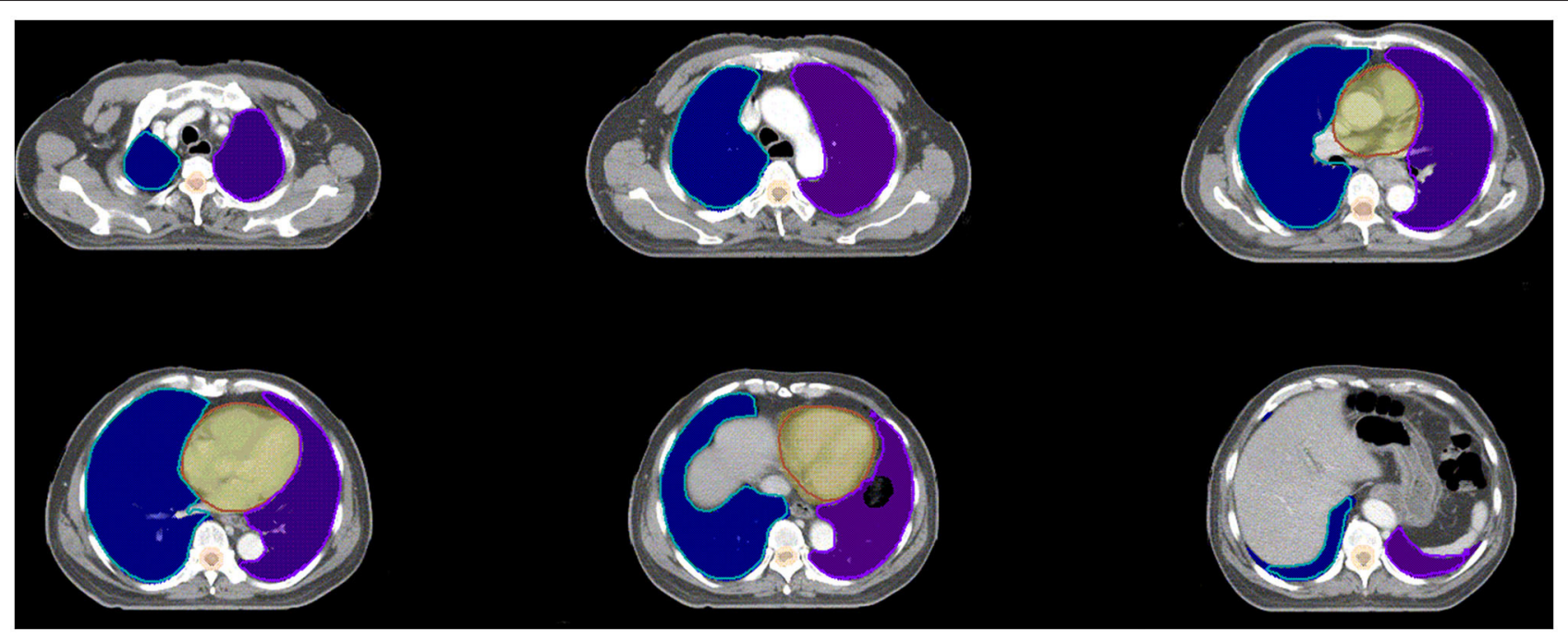

FIGURE 3 | Examples of the segmentation. Color wash: manual segmentation; Line: automatic segmentation.

\section{RESULTS}

\section{Geometric Metrics}

The performance of our deep learning model is shown in Table 2. The MDAs of the left lung, right lung, bilateral lung, heart, spinal cord, spinal cord PRV, left kidney, and right kidney were $0.68 \pm 0.13,0.82 \pm 0.20,0.73 \pm 0.12,1.87 \pm 0.69,0.79 \pm 0.15$, $0.80 \pm 0.15,1.12 \pm 0.31$, and $1.01 \pm 0.29 \mathrm{~mm}$, respectively. The segmentation accuracy values for the left lung, right lung, bilateral lung, heart, spinal cord, spinal cord PRV, left kidney, and right kidney in validation set are shown as follows: DSC: $0.97 \pm 0.01,0.97 \pm 0.01,0.97 \pm 0.01,0.93 \pm 0.03,0.83 \pm 0.03$, $0.91 \pm 0.02,0.93 \pm 0.02$, and $0.93 \pm 0.02$, respectively. It implied that the deep learning model was reliable in automatically delineated OARs for esophageal cancer.

Table 3 shows the mean value and standard deviation of the DSC and MDA, respectively. It also shows that the MDA of the spinal cord and spinal cord PRV was shorter than that of the left lung, right lung, bilateral lung, and heart. The MDAs of the left
TABLE 2 | The geometric characteristic of OARs of validation set.

\begin{tabular}{lcc}
\hline OARs & MDA $(\mathbf{m m})$ & DSC \\
\hline Lung L & $0.68 \pm 0.13$ & $0.97 \pm 0.01$ \\
Lung R & $0.82 \pm 0.20$ & $0.97 \pm 0.01$ \\
Lung all & $0.73 \pm 0.12$ & $0.97 \pm 0.01$ \\
Heart & $1.87 \pm 0.69$ & $0.93 \pm 0.03$ \\
Spinal cord & $0.79 \pm 0.15$ & $0.83 \pm 0.03$ \\
Spinal cord PRV & $0.80 \pm 0.15$ & $0.91 \pm 0.02$ \\
Kidney L & $1.12 \pm 0.31$ & $0.93 \pm 0.02$ \\
Kidney R & $1.01 \pm 0.29$ & $0.93 \pm 0.02$
\end{tabular}

MDA, mean distance to agreement; DSC, Dice similarity coefficient.

lung, right lung, bilateral lung, heart, spinal cord, and spinal cord PRV were $0.82 \pm 0.21,0.90 \pm 0.31,0.85 \pm 0.20,1.74 \pm 0.85$, $0.75 \pm 0.22$, and $0.74 \pm 0.22 \mathrm{~mm}$, respectively. 


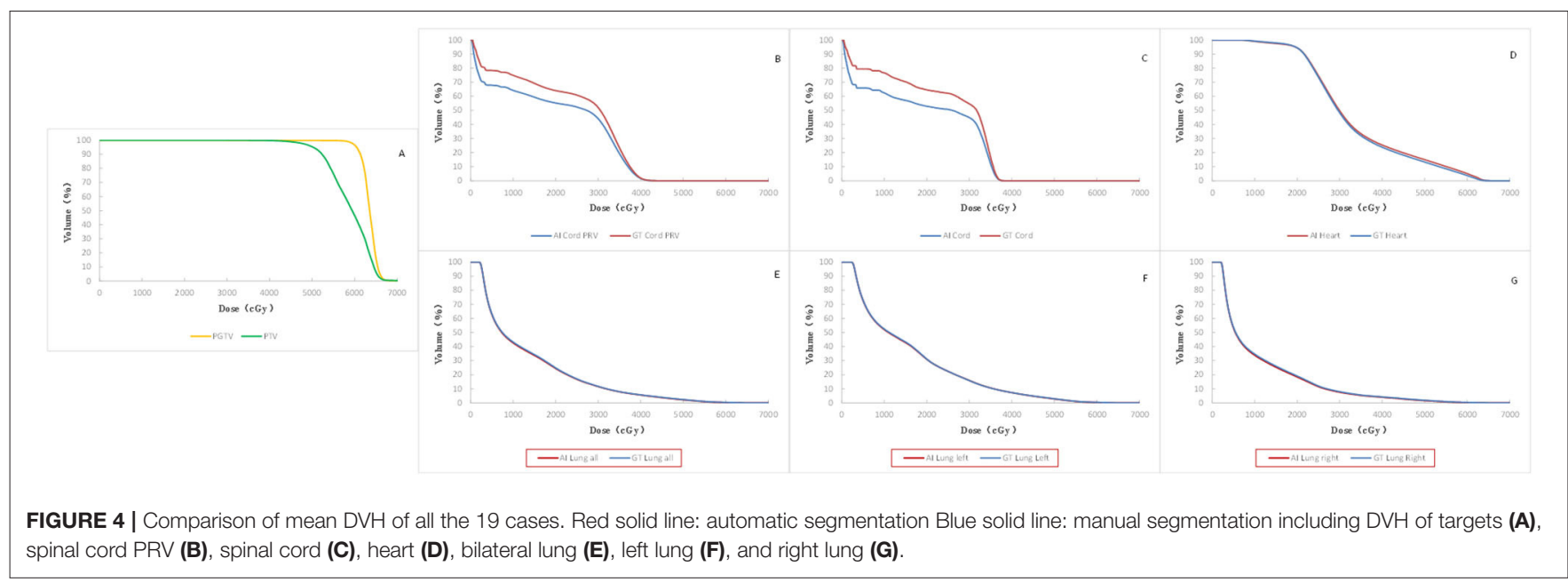

The spinal cord DSC value was $0.84 \pm 0.04$, which was the lowest value in all of six OARs. The OARs including the left lung, right lung, bilateral lung, and heart showed good performance in DSC evaluation. The segmentation accuracy values for the spinal cord PRV, heart, left lung, right lung, and bilateral lung (lung all) are shown as follows: DSC: $0.92 \pm 0.02,0.93 \pm 0.04$, $0.97 \pm 0.01,0.97 \pm 0.01$, and $0.97 \pm 0.01$, correspondingly. Examples of the segmentation results are shown in Figure 3, which illustrates that the segmentation was in good agreement with the manual delineation.

\section{Dosimetric Metrics}

Table 4 shows the paired $t$-test confidence interval of the spinal cord and spinal cord PRV D2\% conversely. The dose difference of spinal cord D2\% between manual and automatic delineations was significant. The V30, V40, and mean dose of the heart were insignificant. All of the corresponding paired $t$-test confidence interval data of the right lung presented were insignificant. By contrast, the $P$-value of the left lung was $<0.001$, except for the $\mathrm{V} 5$ of the left lung. For the bilateral lung, the corresponding V30, $\mathrm{V} 20, \mathrm{~V} 10$, and mean of manual delineation were significantly higher than those of the automatic delineation. V5 of the bilateral lung was insignificant, with interval confidence of 0.44 . Except the V30, V40, and mean of heart, as well as the V5 of right and left lung, most of the dosimetric metrics of manual delineation OARs were found to be relatively significantly higher than automatic delineation OARs. For all of patients' OARs, including spinal cord and lungs, both the manual and automatic delineation plans were able to meet the clinical dose constraints. Only the heart V30 of two patients (\#1: manual: $40.71 \%$, automatic: $41.09 \%$; \#2: manual: $49.56 \%$, automatic: $48.02 \%$ ) could not meet the clinical dose constraints because of their targets close to their heart. However, the heart V30 of these two patients was still variation-acceptable in the clinic.

The mean dose volume histogram curves (Figure 4) of plans with manual and automatic segmentation were close for most of the OARs. Table 4 shows that the maximum dosimetric metrics differences of OARs between manual and automatic delineations were $\Delta \mathrm{D} 2 \%=0.35 \mathrm{~Gy}$ for spinal cord and $\Delta \mathrm{V} 30=0.4 \%$ for
TABLE 3 | The geometric characteristic of OARs between manual and deep learning automatic delineation-based plan.

\begin{tabular}{lcc}
\hline OARs & MDA $(\mathbf{m m})$ & DSC \\
\hline Lung L & $0.82 \pm 0.21$ & $0.97 \pm 0.01$ \\
Lung R & $0.90 \pm 0.31$ & $0.97 \pm 0.01$ \\
Lung all & $0.85 \pm 0.20$ & $0.97 \pm 0.01$ \\
Heart & $1.74 \pm 0.85$ & $0.93 \pm 0.04$ \\
Spinal cord & $0.75 \pm 0.22$ & $0.84 \pm 0.04$ \\
Spinal cord PRV & $0.74 \pm 0.22$ & $0.92 \pm 0.02$ \\
\hline
\end{tabular}

MDA, mean distance to agreement; DSC, Dice similarity coefficient.

bilateral lung, which corresponded with the clinical criteria in this study. The CIs of PTV and PGTV were $0.73 \pm 0.083$ and $0.83 \pm 0.071$, respectively. In addition, The HIs of PTV and PGTV were $0.27 \pm 0.020$ and $0.085 \pm 0.014$, correspondingly.

\section{DISCUSSION}

The geometric results illustrate that the segmentation was in close agreement with the manual delineation when considering the DSC. Because of the lack of relevant reports on automatic delineation in esophageal cancer, we can compare them with thoracic OAR automatic delineation reports. Yang et al. (3) addressed the mean value of DSC in thoracic automatic segmentation. Their data included the left lung, right lung, heart, and spinal cord whose DSC values were $0.956 \pm 0.019$, $0.955 \pm 0.019,0.931 \pm 0.015$, and $0.862 \pm 0.038$, respectively. Dong et al. (5) addressed that the averaged DSC scores for the left lung, right lung, spinal cord, and heart OARs were 0.97, 0.97, 0.90 , and 0.87 , respectively, in 2019 . The lowest DSC value in all of the six OARs is the spinal cord $(0.84 \pm 0.04)$ in our study. The OARs including the spinal cord PRV $(0.92 \pm 0.02)$, left lung (0.97 \pm 0.01$)$, right lung $(0.97 \pm 0.01)$, lung all $(0.97 \pm 0.01)$, and heart $(0.93 \pm 0.04)$ showed good performance in DSC evaluation. Therefore, OARs including the spinal cord, lungs, and heart were 


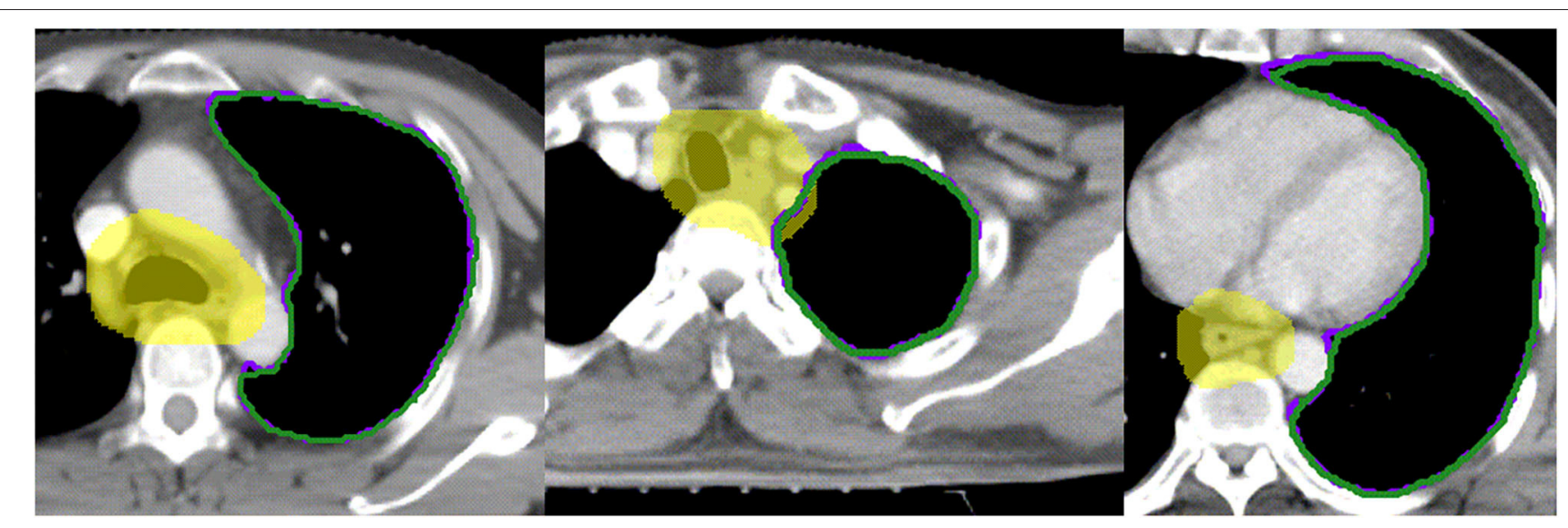

FIGURE 5 | Examples of the segmentation of left hilum. Yellow color wash: PTV; Purple line: manual segmentation; Green line: automatic segmentation.

accurately segmented by the automatically delineated method in this study.

The finding of CIs and HIs above indicate that each radiotherapy plan has good conformity and homogeneity, which can fully meet the clinical requirement. The dosimetric metrics of the spinal cord PRV, heart, and right lung between manual and automatic delineations show no difference in this study. The corresponding $P$-value of the dosimetric metrics between the manual and automatic delineation sets shows an insignificant value that could indicate their equivalent nature. The automatic delineation of the heart and right lung shows a relative equivalent quality in dosimetric metrics when compared with manual delineation.

By contrast, the D2\% of the spinal cord and the mean dose, V10, V20, and V30 of the left and bilateral lungs show significant value $(P<0.05)$. Based on our knowledge, the hilum of lung is a steep dose falloff area in esophageal cancer radiotherapy. As an observation of manually and automatically delineated OARs in Figure 5, the automatically delineated left lung shows a distortion around the left hilum comparing with manual delineation. Therefore, dosimetric metrics of the left and bilateral lungs show significant values in paired $t$ test. The difference of V5 was insignificant for right, left, and bilateral lung. Considering the toxicity of radiotherapy in the lung, Luna et al. (18) reported that the lung V5 (>43.6\%) could predict the presence of radiation pneumonitis consistently. The mean V5 values of manually and automatically delineated bilateral lung were $<43.6 \%$, which implies a lower risk of severe radiation pneumonitis in this study. By the review of studies and radiotherapy guideline, the dose impact is notable in the steep dose fall area (7). The $2 \%$ volume of the spinal cord (manual $1.13 \pm 0.35 \mathrm{cc}$ vs. automatic $1.18 \pm 0.26 \mathrm{cc}$ ) was relatively equal to a voxel of $\mathrm{CT}$ in the planning system (1 $\mathrm{cm} \times 1 \mathrm{~cm} \times 1 \mathrm{~cm})$. The average $2 \%$ volumes of the spinal cord PRV were $4.08 \pm 0.75$ and $4.25 \pm 0.66 \mathrm{cc}$ for manual and automatic delineations, respectively. D2\% of the spinal cord was relatively equal to the point dose in radiotherapy planning. Therefore, the $\mathrm{D} 2 \%$ of the spinal cord shows significant value
TABLE 4 | The paired $t$-test outcome of the dosimetric characteristic of OARs between manual and deep learning automatic delineation-based plan.

\begin{tabular}{|c|c|c|c|c|}
\hline Dosimetric metrics & & GT & Al & $P$-value \\
\hline Spinal cord & D2\% (Gy) & $36.08 \pm 0.41$ & $35.73 \pm 0.41$ & $<0.01$ \\
\hline Spinal cord PRV & D2\% (Gy) & $40.25 \pm 0.43$ & $40.42 \pm 0.30$ & 0.55 \\
\hline \multirow[t]{3}{*}{ Heart } & V30 (\%) & $28.60 \pm 4.06$ & $28.70 \pm 4.09$ & 0.87 \\
\hline & V40 (\%) & $14.68 \pm 2.19$ & $15.00 \pm 2.28$ & 0.48 \\
\hline & Mean (Gy) & $20.54 \pm 2.71$ & $20.64 \pm 2.76$ & 0.65 \\
\hline \multirow[t]{5}{*}{ Lung all } & V30 (\%) & $8.63 \pm 2.69$ & $8.23 \pm 2.73$ & 0.02 \\
\hline & V20 (\%) & $15.81 \pm 4.95$ & $15.63 \pm 4.99$ & $<0.01$ \\
\hline & V10 (\%) & $26.47 \pm 8.27$ & $26.28 \pm 8.28$ & 0.04 \\
\hline & V5 (\%) & $41.05 \pm 12.76$ & $41.48 \pm 13.18$ & 0.44 \\
\hline & Mean (Gy) & $9.26 \pm 2.55$ & $9.21 \pm 2.57$ & 0.04 \\
\hline \multirow[t]{5}{*}{ Lung L } & V30 (\%) & $10.24 \pm 4.86$ & $10.01 \pm 4.95$ & $<0.01$ \\
\hline & V20 (\%) & $18.55 \pm 8.28$ & $18.28 \pm 8.38$ & $<0.01$ \\
\hline & V10 (\%) & $30.53 \pm 12.02$ & $30.31 \pm 12.07$ & 0.04 \\
\hline & V5 (\%) & $45.84 \pm 16.26$ & $45.88 \pm 16.45$ & 0.73 \\
\hline & Mean (Gy) & $10.31 \pm 3.68$ & $10.21 \pm 3.71$ & $<0.01$ \\
\hline \multirow[t]{5}{*}{ Lung R } & V30 (\%) & $7.31 \pm 3.75$ & $7.32 \pm 3.79$ & 0.90 \\
\hline & V20 (\%) & $13.55 \pm 5.35$ & $13.45 \pm 5.28$ & 0.41 \\
\hline & V10 (\%) & $23.11 \pm 7.83$ & $22.97 \pm 1.76$ & 0.42 \\
\hline & V5 (\%) & $37.04 \pm 11.71$ & $37.01 \pm 11.74$ & 0.89 \\
\hline & Mean (Gy) & $8.40 \pm 2.51$ & $8.38 \pm 2.51$ & 0.75 \\
\hline
\end{tabular}

$(P<0.05)$. The dosimetric metrics of spinal cord PRV is more important in this study because the PRV is recommended for the structures of the nervous system including the spinal cord $(11,19)$.

Although the dose differences of the spinal cord, left lung, and bilateral lung are significant, their absolute difference is small and acceptable for clinical use. Table 4 shows that the maximum dosimetric metrics differences of OARs between manual and automatic delineations are $<1$ Gy (spinal cord, $\Delta \mathrm{D} 2 \%=0.35 \mathrm{~Gy}$ ) and $1 \%$ (bilateral lung, 
$\Delta \mathrm{V} 30=0.4 \%)$. The dose difference and volume difference of OARs had no impact on the radiation toxicity of each OARs, because the OARs of both manual and automatic delineations do not approach their maximum tolerance in this study (8). Chicas-Sett et al. (20) reported that the manual delineation also depends on intraobserver or interobserver deviations, which leads to dosimetric difference and organsparing failure.

As shown in the results, the dosimetric metrics of manual delineation OARs were found to be relatively significantly higher or lower than automatic delineation OARs. However, the dosimetric metrics of manually delineated OARs for each patient did not show a directional higher or lower trend than automatic delineation OARs. This result implies that the dosimetric metrics of manual and automatic delineation methods conform to Gaussian distribution, which had been proved in paired $t$-test.

Although the deep learning segmentation model shows outperformance, there are still limitations to this study. In order to improve the performance of automatic delineation model, larger training data are recommended in future work. Further, three-dimensional radiography information will be valuable in the architecture of deep learning model. As shown in Figure 5, the automatically delineated left lung illustrates a distortion around the left hilum. This limitation might be overcome with the combination of threshold method and automatic delineation.

\section{CONCLUSION}

The findings of this study showed that the geometric evaluation between manual and automatic delineations was not enough in clinical applications. Dosimetric metrics were proposed to assess the automatic delineation in radiotherapy planning of esophageal cancer. Based on the dosimetric evaluation in this study, the

\section{REFERENCES}

1. Liang S, Tang F, Huang X, Yang K, Zhong T, Hu R, et al. Deep-learning-based detection and segmentation of organs at risk in nasopharyngeal carcinoma computed tomographic images for radiotherapy planning. Eur Radiol. (2019) 29:1961-7. doi: 10.1007/s00330-018-5748-9

2. Ahn SH, Yeo AU, Kim KH, Kim C, Goh Y, Cho S, et al. Comparative clinical evaluation of atlas and deep-learning-based autosegmentation of organ structures in liver cancer. Radiat Oncol. (2019) 14:213. doi: 10.1186/s13014-019-1392-z

3. Yang J, Veeraraghavan H, Armato SG 3rd, Farahani K, Kirby JS, KalpathyKramer J, et al. Autosegmentation for thoracic radiation treatment planning: a grand challenge at AAPM (2017). Med Phys. (2018) 45:456881. doi: $10.1002 / \mathrm{mp} .13141$

4. Lustberg T, Soest JV, Gooding M, Peressutti D, Dekker A. Clinical evaluation of atlas and deep learning based automatic contouring for lung cancer. Radiother Oncol. (2017) 126:312-7. doi: 10.1016/j.radonc.2017.11.012

5. Dong X, Lei Y, Wang T, Thomas M, Tang L, Curran WJ, et al. Automatic multiorgan segmentation in thorax CT images using U-Net-GAN. Med Phys. (2019) 46:2157-68. doi: 10.1002/mp. 13458

6. Vinod SK, Jameson MG, Min M, Holloway LC. Uncertainties in volume delineation in radiation oncology: a systematic review manual delineation for esophageal cancer radiotherapy can be substituted by automatic delineation.

\section{DATA AVAILABILITY STATEMENT}

The datasets presented in this article are not readily available because of privacy requirements of the hospital. Requests to access the datasets should be directed to the corresponding author.

\section{ETHICS STATEMENT}

This study was carried out in accordance with the Declaration of Helsinki and was approved with exemption from informed consent by the Independent Ethics Committee of Cancer Hospital, Chinese Academy of Medical Sciences.

\section{AUTHOR CONTRIBUTIONS}

All authors discussed and conceived of the study design. JZ and $\mathrm{XC}$ trained the deep learning models, performed data analysis, and drafted the manuscript. BY, NB, and TZ helped to collect the data and evaluate radiotherapy planning. JD and KM guided the study and participated in discussions and preparation of the manuscript. All authors read, discussed, and approved the final manuscript.

\section{FUNDING}

This work was supported by the National Natural Science Foundation of China (11875320 and 11975313), the Beijing Municipal Science and Technology Commission (Z181100001918002), the Beijing Hope Run Special Fund of Cancer Foundation of China (LC2019B06 and LC2018A14), and Beijing Nova Program (Z201100006820058). and recommendations for future studies. Radiother Oncol. (2016) 121:169-79. doi: 10.1016/j.radonc.2016.09.009

7. Fung NTC, Hung WM, Sze CK, Lee $\mathrm{MCH}$, Ng WT. Automatic segmentation for adaptive planning in nasopharyngeal carcinoma IMRT: Time, geometrical, dosimetric analysis. Med Dosimetry. (2019) 45:6065. doi: 10.1016/j.meddos.2019.06.002

8. Bradley J, Schild S, Bogart J, Dobelbower M, Choy H, Adjei A. RTOG 0617/NCCTG N0628/CALGB 30609/ECOG R0617: A Randomized Phase III Comparison of Standard Dose (60Gy) versus High-Dose (74Gy) Conformal Radiotherapy With Concurrent and Consolidation Carboplatin/Paclitaxel \pm Cetuximab (IND\# 103444) in Patients With Stage IIIa/IIIb Non-Small Cell Lung Cancer. Available online at: http://www.rtog. org (accessed October 30, 2009).

9. Kong F, Machtay M, Bradley J, Ten Haken R, Xiao Y, Matuszak M, et al. RTOG 1106/ACRIN 6697: Randomized Phase II Trial of Individualized Adaptive Radiotherapy Using during Treatment FDG-PECT T, and Modern Technology in Locally Advanced Non-Small Lung Cancer (NSCLC) (2012).

10. Men K, Zhang $\mathrm{T}$, Chen $\mathrm{X}$, Chen $\mathrm{B}$, Tang $\mathrm{Y}$, Wang $\mathrm{S}$, et al. Fully automatic and robust segmentation of the clinical target volume for radiotherapy of breast cancer using big data and deep learning. Phys Med. (2018) 50:13-9. doi: 10.1016/j.ejmp.2018. 05.006 
11. Gregoire V, Mackie T, Neve W. Prescribing, recording, and reporting photonbeam intensity-modulated radiation therapy (IMRT). J Int Commission Radiat Units Meas. (2010) 10:1-106. doi: 10.1093/jicru/ndq002

12. Speight R, Karakaya E, Prestwich R, Sen M, Lindsay R, Harding R, et al. Evaluation of atlas based auto-segmentation for head and neck target volume delineation in adaptive/replan IMRT. J Phys Conf Ser. (2014) 489:012060. doi: 10.1088/1742-6596/489/1/012060

13. Hanna G, Hounsell A, O'Sullivan J. Geometrical analysis of radiotherapy target volume delineation: a systematic review of reported comparison methods. Clin Oncol. (2010) 22:515-25. doi: 10.1016/j.clon.2010. 05.006

14. Hosseini M-P, Nazem-Zadeh MR, Pompili D, Soltanian-Zadeh H. Statistical validation of automatic methods for hippocampus segmentation in MR images of epileptic patients. Conf Proc IEEE Eng Med Biol Soc. (2014) 2014:4707-10. doi: 10.1109/EMBC.2014.6944675

15. Franco P, Arcadipane F, Trino E, Gallio E, Martini S, Iorio GC, et al. Variability of clinical target volume delineation for rectal cancer patients planned for neoadjuvant radiotherapy with the aid of the platform Anatome. Clin Transl Radiat Oncol. (2018) 11:33-9. doi: 10.1016/j.ctro.2018. 06.002

16. Paddick I, A simple scoring ratio to index the conformity of radiosurgical treatment plans. J Neurosurg. (2000) 93:21922. doi: 10.3171/jns.2000.93.supplement_3.0219

17. Yan L, Xu Y, Chen X, Xie X, Liang B, Dai J. A new homogeneity index definition for evaluation of radiotherapy plans. J Appl Clin Med Phys. (2019) 20:50-6. doi: 10.1002/acm2.12739
18. Luna JM, Chao H-H, Diffenderfer ES, Valdes G, Chinniah C, Ma G, et al. Predicting radiation pneumonitis in locally advanced stage II-III non-small cell lung cancer using machine learning. Radiother Oncol. (2019) 133:10612. doi: 10.1016/j.radonc.2019.01.003

19. Wilke L, Andratschke N, Blanck O, Brunner TB, Combs SE, Grosu A-L, et al. ICRU report 91 on prescribing, recording, and reporting of stereotactic treatments with small photon beams. Strahlenther Onkol. (2019) 195:193-8. doi: 10.1007/s00066-0181416-x

20. Chicas-Sett R, Celada-Alvarez F, Roldan S, Rodriguez-Villalba S, Santos-Olias M, Soler-Catalan $\mathrm{P}$, et al. Interobserver variability in rectum contouring in high-dose-rate brachytherapy for prostate cancer: a multi-institutional prospective analysis. Brachytherapy. (2018) 17:208-13. doi: 10.1016/j.brachy.2017.09.015

Conflict of Interest: The authors declare that the research was conducted in the absence of any commercial or financial relationships that could be construed as a potential conflict of interest.

Copyright (C) 2020 Zhu, Chen, Yang, Bi, Zhang, Men and Dai. This is an open-access article distributed under the terms of the Creative Commons Attribution License (CC $B Y)$. The use, distribution or reproduction in other forums is permitted, provided the original author(s) and the copyright owner(s) are credited and that the original publication in this journal is cited, in accordance with accepted academic practice. No use, distribution or reproduction is permitted which does not comply with these terms. 